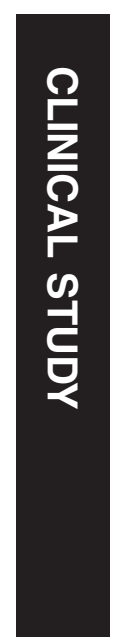

\title{
Peripheral lamina cribrosa depth in primary open-angle glaucoma: a swept- source optical coherence tomography study of lamina cribrosa
}

Department of

Ophthalmology, Seoul National University Hospital, Seoul National University College of Medicine, Seoul, Korea

Correspondence: KH Park, Department of Ophthalmology, Seoul National University College of Medicine, 101 Daehakro, Jongno-gu, Seoul, 110-744, Korea

Tel: +82 22072 3172;

Fax: +82 27413187

E-mail: kihopark@snu.ac.kr

Received: 24 February 2015 Accepted in revised form: 27 July 2015

Published online:

21 August 2015

Meeting Presentation: Poster presentation at AAO annual meeting 2014, Chicago. (PO382)

\begin{abstract}
Purpose To investigate peripheral lamina cribrosa depth (PLCD) and its verticalhorizontal difference in eyes with primary open-angle glaucoma (POAG).

Methods Patients with POAG ( $n=90$ eyes) and age-matched healthy individuals $(n=90$ eyes) underwent swept-source optical coherence tomography (SS-OCT) scans centered at the optic discs. The PLCD was defined as the vertical distance between the most peripheral visible end of anterior lamina cribrosa (LC) surface and the reference plane connecting the Bruch's membrane openings. The PLCD in each quadrant region and the vertical-horizontal PLCD difference were compared between the POAG and healthy eyes. The clinical factors associated with increased PLCD were evaluated. Results The PLCD was significantly larger in the POAG eyes than the control eyes at the horizontal $(P=0.034)$ and vertical $(P=0.001)$ meridians. The vertical PLCD was significantly larger than the horizontal PLCD, both in the POAG eyes $(P<0.001)$ and in the control eyes $(P=0.003)$. However, the vertical-horizontal PLCD difference was significantly larger in the POAG eyes $(47 \pm 60 \mu \mathrm{m})$ than in the control eyes $(18 \pm 54 \mu \mathrm{m}, P=0.001)$. Multivariate regression showed a significant association of male gender $(P=0.005)$, increased baseline IOP $(P=0.043)$, and decreased MD of VF $(P=0.025)$ with increased PLCD. Conclusions The peripheral LC was displaced more posteriorly in the POAG eyes compared with the age-matched healthy eyes. In the POAG eyes, the peripheral LC was displaced more posteriorly at the vertical
\end{abstract}

YW Kim, DW Kim, JW Jeoung, DM Kim and KH Park meridian than at the horizontal meridian. The peripheral LC in the vertical meridian might have increased IOP-related strain (deformation) compared with horizontal meridian in glaucomatous eyes.

Eye (2015) 29, 1368-1374; doi:10.1038/eye.2015.162; published online 21 August 2015

Introduction

The lamina cribrosa (LC), a multi-layered collagenous sieve-like structure at the optic nerve head, is presumed to be the primary site of axonal injury in glaucoma. ${ }^{1-7}$ Under the biomechanical paradigm for glaucoma pathogenesis, the influence of increased intraocular pressure (IOP) on the LC has been widely elucidated in experimental glaucoma animal models. ${ }^{7-12}$ Early experimental glaucoma monkey eyes, for example, showed posterior migration of anterior and posterior laminar insertion. ${ }^{12}$ Posterior displacement of laminar insertion has been confirmed in autopsied and enucleated human eyes with advanced glaucoma. ${ }^{3}$ Recently, posterior displacement of anterior laminar insertion has been investigated in in vivo human eyes using swept-source optical coherence tomography (SS-OCT). ${ }^{13}$

Human autopsy findings have demonstrated that the superior and inferior regions of the LC have larger pore size and lower connective tissues than do the nasal and temporal quadrants. ${ }^{2,5,14,15}$ This anatomical difference is more remarkable in the peripheral than in the central region of the LC. ${ }^{14}$ The regional variance in laminar pore and beam size has been suggested to be related with glaucomatous optic 
nerve damage commonly seen in superior and inferior retinal nerve fibers. ${ }^{2,4-6,15}$ Mathematical models have predicted that local stresses and strains on the LC are dependent on its microarchitecture. ${ }^{16}$ The influence of biomechanical stress on LC posterior deformation, then, might differ according to the vertical-horizontal meridian.

We hypothesized that (i) peripheral LC is located more posteriorly in eyes with primary open-angle glaucoma (POAG) than in age-matched healthy eyes, and that (ii) the vertical-horizontal difference of PLCD is larger in eyes with POAG than in healthy eyes. The purpose of the present study was to investigate the peripheral LC location and its vertical-horizontal difference in POAG and age-matched healthy eyes.

\section{Materials and methods}

The participants of the present study comprised glaucoma patients and healthy individuals from the Swept-Source Optical Coherence Tomography Study of Lamina Cribrosa (SOS-LC), an ongoing prospective study at Seoul National University Hospital (SNUH). The glaucoma patients had visited the SNUH glaucoma clinic, and the age-matched healthy individuals had visited the SNUH outpatient clinic for regular ocular check-ups (eg, dry eye, cataract). The present study was approved by the Seoul National University Hospital Institutional Review Board and followed the tenets of the Declaration of Helsinki.

\section{Study subjects}

All of the subjects underwent complete ophthalmic examinations including visual acuity assessment, slitlamp biomicroscopy, gonioscopy, Goldmann applanation tonometry, refraction, dilated fundus examination, disc stereophotography and red-free fundus photography by digital fundus camera (VX-10, Tokyo, Japan), and standard automated perimetry (Humphrey C 30-2 SITA-Standard visual field (VF); Carl Zeiss Meditec, Inc., Dublin, CA, USA). The central corneal thickness

(Pocket II; Quantel Medical, Clermont-Ferrand, France) and axial length (AXIS-II Ultrasonic Biometer; Quantel Medical S.A., Bozeman, MT, USA) were measured. The average retinal nerve fiber layer (RNFL) thickness and the disc area were measured by Cirrus HD-OCT (Carl-Zeiss Meditec) via $200 \times 200$ optic disc cube scan. POAG was defined as the presence of glaucomatous optic disc change including loss of neuroretinal rim with vertical CDR $>0.7$ and/or focal notching with RNFL defect and/or asymmetry of CDR between eyes $>0.2$, RNFL defect on disc stereophotography and red-free fundus photography, a glaucomatous VF defect, and an open angle confirmed by gonioscopic examination.

A glaucomatous VF defect was defined as (i) glaucoma hemifield test values outside the normal limits or (ii) three or more abnormal points with a probability of $P<0.05$, of which at least one point has a pattern deviation of $P<0.01$, or (iii) a pattern standard deviation of $P<0.05$. Those VF defects were confirmed on two consecutive reliable tests (fixation loss rate $\leq 20 \%$, false-positive and false negative error rates $\leq 25 \%$ ). The baseline IOP was defined as the mean of the two measurements before IOPlowering treatment. The age-matched healthy individuals showed no abnormalities in disc stereophotography, redfree fundus photography, and standard automated perimetry.

The present study excluded subjects with (i) a history of intraocular surgery including glaucoma surgery, (ii) a history of intraocular disease (eg, proliferative diabetic retinopathy, retinal vein occlusion), (iii) an axial length longer than $27 \mathrm{~mm}$, (iv) severely tilted optic discs with nasalization of the central vessels, or (v) SS-OCT scans with artifacts, vascular shadowing, or peripheral focal LC defects obscuring the peripheral LC. ${ }^{17,18}$

\section{SS-OCT imaging of optic disc}

All of the participants underwent scanning with the DRI OCT-1 Atlantis 3D SS-OCT device (Topcon Medical Systems, Oakland, NJ, USA). This system operates at a speed of 100000 A-scans/s using a laser of $1050 \mathrm{~nm}$ wavelength, and has been claimed to have an axial resolution of $8 \mu \mathrm{m}$ and lateral resolution of $20 \mu \mathrm{m}$. The OCT examination was performed in a dark room and with eyes dilated. The scan was performed for both eyes, one of which was then randomly selected for the analysis.

In acquiring the LC images, five line cross scans (five lines vertical and five lines horizontal) centered at the optic disc with 0.25 -mm spacing between the crosslines and a $6.0 \mathrm{~mm}$ scan width, were performed. A total of 32 A-scans were averaged for each line of five cross-lines.

\section{Measurements of peripheral LC depth}

In the present study, the PLCD was defined as the vertical distance between the most peripheral visible end of anterior LC surface and the reference plane connecting the Bruch's membrane openings (Figure 1). Each PLCD was measured in both horizontal (temporal and nasal) and vertical (superior and inferior) scans. The central three of the five cross-lines at each meridian were selected for the measurements, and the means of the measured values were analyzed. The horizontal PLCD was the mean of the temporal and nasal PLCD, and the vertical PLCD was the mean of the superior and inferior PLCD. The overall PLCD was defined as the mean of the horizontal and vertical PLCD. The measurements were performed by an 
experienced ophthalmologist (YWK) masked to each subjects' clinical information.

\section{Statistical analysis}

To evaluate the intraobserver and interobserver reproducibility of the PLCD measurement, two observers (YWK and DWK), in a masked manner, performed measurements in 20 randomly selected SS-OCT B-scans. The analysis was based on three independent series of re-evaluations; the absolute agreement of a single observer's measurement and the mean of all three measurements of the two observers was calculated with the intraclass correlation coefficient (ICC) obtained from a two-way mixed-effect model.

The continuous variables were compared using a student $t$-test or a paired $t$-test. The categoric variables were compared using a chi-square test. The general linear model was used to determine the factors (age, gender, diabetes mellitus, hypertension, baseline IOP, central corneal thickness, axial length, mean deviation (MD) of VF, and average RNFL thickness) associated with increased overall PLCD, first with a univariate model,

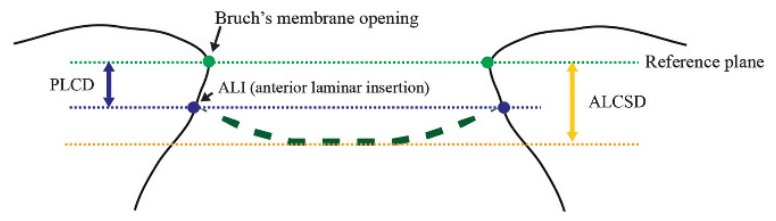

Figure 1 Schematic diagram showing measurement of PLCD. The PLCD was defined as the vertical distance between the most peripheral visible end of the anterior LC surface and the reference plane connecting the Bruch's membrane openings. Anterior laminar cribrosa depth, or anterior laminar cribrosa surface depth (ALCSD), is the vertical distance between the reference plane and the anterior LC surface. and then with a multivariate model that included the univariate model variables with $P<0.05$. Statistical analyses were performed with the Statistical Package for Social Sciences version 21.0 for Windows (SPSS, Inc., Chicago, IL, USA). The data were obtained, and are presented in this paper, as mean \pm standard deviations, and the level of statistical significance was set at $P<0.05$.

\section{Results}

\section{Baseline characteristics}

The present study included 90 eyes of 90 POAG patients and 90 eyes of 90 age-matched healthy individuals. There were no differences in gender, underlying disease (ie, diabetes and hypertension), refraction, disc area, axial length, or central corneal thickness between the two groups. The baseline IOP was significantly higher in the POAG group $(16.1 \pm 3.4 \mathrm{~mm} \mathrm{Hg}$ vs $13.3 \pm 3.1 \mathrm{~mm} \mathrm{Hg}$, $P<0.001)$. As the POAG group had been under intensive treatment for IOP control, their IOP at the examination was significantly lower than that of the age-matched healthy control group $(12.5 \pm 2.2 \mathrm{~mm} \mathrm{Hg}$ vs $13.3 \pm 2.8 \mathrm{~mm} \mathrm{Hg}, P=0.021)$. The average treatment period from the baseline examination to the time of OCT imaging was $4.3 \pm 4.1$ years ( 1 month -15.8 years). The MD of VF and the average RNFL thickness were significantly lower in the POAG group than in the age-matched healthy control group (all $P<0.001$, Table 1 ).

\section{Peripheral LC depth}

The central three of five cross-line scans (total: 540 horizontal and 540 vertical scans of 180 subjects) were selected, of which the means were used for the analysis. Thirty-two (32) scans (20 horizontal, 12 vertical) were

Table 1 Patient demographics

\begin{tabular}{|c|c|c|c|}
\hline Variable & POAG ( $\mathrm{n}=90$ eyes $)$ & Healthy ( $\mathrm{n}=90$ eyes) & P-value \\
\hline Age, year & $61.5 \pm 10.2$ & $61.4 \pm 10.8$ & $0.94^{\mathrm{a}}$ \\
\hline Female, $n(\%)$ & $44(48.9)$ & $53(58.9)$ & $0.23^{\mathrm{b}}$ \\
\hline Hypertension, $n(\%)$ & $21(23.3)$ & $24(26.7)$ & $0.73^{\mathrm{b}}$ \\
\hline Diabetic mellitus, $n(\%)$ & $10(11.1)$ & $15(16.7)$ & $0.39^{\mathrm{b}}$ \\
\hline IOP at examination, $\mathrm{mm} \mathrm{Hg}$ & $12.5 \pm 2.2$ & $13.3 \pm 2.8$ & $0.021^{\mathrm{a}}$ \\
\hline Baseline IOP, $\mathrm{mm} \mathrm{Hg}$ & $16.1 \pm 3.4$ & $13.3 \pm 3.1$ & $<0.001$ \\
\hline SE, D & $-0.9 \pm 2.5$ & $-0.7 \pm 2.8$ & $0.67^{\mathrm{a}}$ \\
\hline $\mathrm{AXL}, \mathrm{mm}$ & $24.0 \pm 1.2$ & $24.0 \pm 1.6$ & $0.82^{\mathrm{a}}$ \\
\hline Central corneal thickness, $\mu \mathrm{m}$ & $533.2 \pm 30.1$ & $541.2 \pm 29.8$ & $0.11^{\mathrm{a}}$ \\
\hline $\mathrm{MD}, \mathrm{dB}$ & $-5.29 \pm 6.19$ & $-0.77 \pm 2.03$ & $<0.001^{a}$ \\
\hline Average RNFL thickness, $\mu \mathrm{m}$ & $74.0 \pm 11.6$ & $89.5 \pm 9.3$ & $<0.001^{\mathrm{a}}$ \\
\hline Disc area, $\mathrm{mm}^{2}$ & $2.08 \pm 0.46$ & $2.14 \pm 0.43$ & 0.39 \\
\hline
\end{tabular}

Abbreviations: AXL, axial length; $\mathrm{D}$, diopter; IOP, intraocular pressure; $\mathrm{MD}$, mean deviation; POAG, primary open-angle glaucoma; $\mathrm{SE}$, spherical equivalence.

${ }^{\text {a }}$ Comparison performed using student $t$-test.

${ }^{\mathrm{b}}$ Comparison performed using chi-square test.

Mean \pm standard deviation, Statistically significant values are shown in bold. 
excluded owing to poor OCT scan quality (ie, severe vascular shadowing) that did not allow clear visualization of the peripheral LC. Therefore, a total of 520 horizontal scans and 528 vertical scans were included in the analysis. Horizontal PLCD measurement by the two observers showed excellent intraobsever (ICC $=0.993$ and 95\% confidence interval $(\mathrm{CI})=0.965-0.999$ for observer 1 ; ICC $=0.998$ and $95 \% \mathrm{CI}=0.993-0.999$ for observer 2 ) and interobserver $(\mathrm{ICC}=0.956$ and $95 \% \mathrm{CI}=0.911-0.978$ ) reproducibility (all $P<0.001$ ). Vertical PLCD measurement by the two observers also exhibited excellent intraobserver (ICC $=0.978$ and 95\% CI $=0.896-$ 0.998 for observer 1 ; ICC $=0.951$ and $95 \% \mathrm{CI}=0.804-0.988$ for observer 2) and interobserver (ICC $=0.964$ and $95 \%$ $\mathrm{CI}=0.928-0.982$ ) reproducibility (all $P<0.001$ ).

The PLCD was significantly larger in the POAG eyes than in the age-matched healthy eyes at the horizontal $(382.6 \pm 155.3 \mu \mathrm{m}$ vs $339.5 \pm 111.6 \mu \mathrm{m}, P=0.034)$ and vertical $(429.9 \pm 159.0 \mu \mathrm{m}$ vs $357.0 \pm 115.6 \mu \mathrm{m}, P=0.001)$ meridians. The temporal $(361.3 \pm 149.6 \mu \mathrm{m} v \mathrm{~s}$ $317.1 \pm 110.9 \mu \mathrm{m}, P=0.025)$, superior $(451.5 \pm 163.4 \mu \mathrm{m} v \mathrm{~s}$ $379.1 \pm 123.2 \mu \mathrm{m}, P=0.001)$, and inferior $(408.4 \pm 159.2 \mu \mathrm{m}$ vs $335.0 \pm 113.1 \mu \mathrm{m}, P<0.001)$ PLCD were significantly larger in the POAG eyes than in the age-matched healthy eyes (Figure 2, Table 2). The nasal PLCD was larger in the POAG eyes $(403.9 \pm 165.5 \mu \mathrm{m})$ than in the age-matched healthy eyes $(362.0 \pm 120.3 \mu \mathrm{m})$ but showed only marginal significance $(P=0.054)$.

\section{Vertical-horizontal PLCD difference}

The vertical PLCD was significantly larger than the horizontal PLCD, both in the POAG eyes $(429.9 \pm 159.0 \mu \mathrm{m}$ vs $382.6 \pm 155.3 \mu \mathrm{m}, P<0.001)$ and in the age-matched healthy eyes $(357.0 \pm 115.6 \mu \mathrm{m}$ vs $339.5 \pm 111.6 \mu \mathrm{m}, P=0.003$ ) (Table 3). However, the vertical-horizontal PLCD difference was significantly larger in the POAG eyes $(47.3 \pm 59.6 \mu \mathrm{m})$ than in the age-matched healthy eyes $(17.5 \pm 53.9 \mu \mathrm{m}, P=0.001)$.

\section{Factors associated with increased overall PLCD}

In the univariate analysis results, the factors associated with increased overall PLCD were male gender $(P=0.017)$, increased baseline IOP $(P=0.036)$, decreased MD of VF $(P=0.002)$, and decreased average RNFL thickness $(P=0.009)$. The variables that showed significance at $P<0.05$, namely male gender, baseline

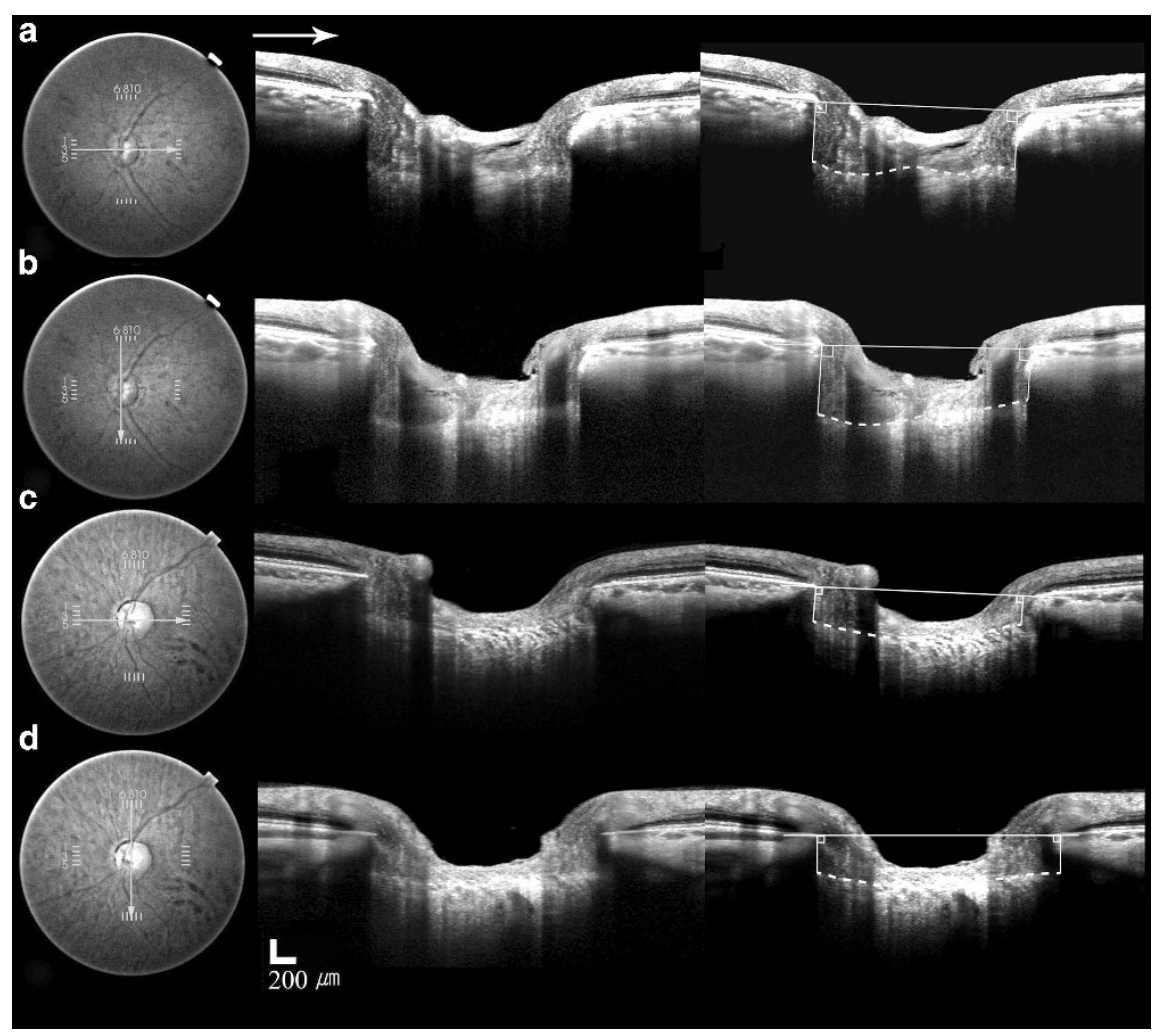

Figure 2 PLCD in POAG and healthy eyes. Demonstration of horizontal and vertical optic disc scans of a POAG eye (a, b) and a healthy eye (c, d) with SS-OCT. The image delineated with yellow guidelines is the same as that depicted on the left side. In POAG eye, the horizontal PLCD was $488.0 \mu \mathrm{m}$ (a), and the vertical PLCD was $546.2 \mu \mathrm{m}$ (b). In healthy eye, the horizontal PLCD was $304.2 \mu \mathrm{m}$ (c), and the vertical PLCD was $309.2 \mu \mathrm{m}$ (d). The full colour version of this figure is available at EYE online. 
IOP, MD of VF, and average RNFL thickness, were included in the multivariate model. To avoid the interaction between the MD of VF and average RNFL thickness, the linear regression analysis was performed separately for these two variables. In the multivariate analysis model 1 , male gender (beta $=83.022, P=0.005$ ), baseline IOP (beta $=9.620, P=0.043$ ), and MD of VF (beta $=-5.479, P=0.025$ ) were significantly associated with increased overall PLCD. In the multivariate analysis model 2, male gender (beta $=62.111, P=0.023$ ) was significantly associated with increased overall PLCD (Supplementary Table 4, see supplementary file).

\section{Discussion}

The present study demonstrated that the vertical peripheral LC is located more posteriorly than the horizontal peripheral LC, in both the POAG and age-matched healthy eyes, where the vertical-horizontal PLCD difference was significantly larger in the POAG eyes.

Park et al ${ }^{19}$ reported that, in healthy eyes measured by EDI-OCT, the anterior laminar insertion was located more posteriorly in the superior and inferior than in the nasal and temporal regions. Their subsequent study found a more posterior location of central and mid-peripheral LC

Table 2 Comparison of peripheral lamina cribrosa depth (PLCD) between primary open-angle glaucoma (POAG) and healthy control eyes

\begin{tabular}{|c|c|c|c|}
\hline$P L C D, \mu m$ & POAG $(\mathrm{n}=90$ eyes $)$ & Healthy $(\mathrm{n}=90$ eyes $)$ & P-value \\
\hline Horizontal & $382.6 \pm 155.3$ & & \\
\hline emporal & $361.3 \pm 149.6$ & 0.9 & $0.025^{a}$ \\
\hline Tasal & 4 & & $0.054^{\mathrm{a}}$ \\
\hline ertical & 429 & 15.6 & $0.001^{\mathrm{a}}$ \\
\hline uperior & $451.5 \pm$ & & $0.001^{\mathrm{a}}$ \\
\hline Inferior & $408.4 \pm 159.2$ & $335.0 \pm 113.1$ & $<0.001^{\mathrm{a}}$ \\
\hline \multicolumn{4}{|c|}{$\begin{array}{l}\text { Abbreviations: PLCD, peripheral lamina cribrosa depth, defined as } \\
\text { vertical distance between most peripheral visible end of anterior lamina } \\
\text { cribrosa surface and reference plane of Bruch's membrane opening; } \\
\text { POAG, primary open-angle glaucoma. } \\
\text { a Comparison performed using student } t \text {-test. } \\
\text { Mean } \pm \text { standard deviation, statistically significant values are shown in bold. }\end{array}$} \\
\hline
\end{tabular}

in glaucomatous than in healthy eyes. ${ }^{20}$ The present data consistently exhibited, via SS-OCT scans, verticalhorizontal PLCD difference in healthy eyes. Further, the present study explored, for the first time using SS-OCT, the increased vertical-horizontal PLCD difference in POAG eyes compared with age-matched healthy eyes. The present finding can be explained by following speculations: Previous histological studies reported the larger pore sizes and lower connective tissues in the superior and inferior regions than in the nasal and temporal regions of the LC. 2,4-6,14,15 This regional structural difference might have resulted in varied IOPrelated strain (deformation) by the vertical-horizontal meridian for a given level of IOP-related stress (force).

In the present multivariate analysis, baseline IOP showed a positive association with the overall PLCD in the POAG eyes. Yang et al ${ }^{12}$ correlated posterior migration of anterior and posterior laminar insertion with increasing IOP in early experimental glaucoma monkey eyes. The present data possibly support previous experimental animal studies that investigated LC posterior displacement with increasing IOP. As the POAG patients were under intensive IOP-lowering management in the present study, the IOP on examination was even lower in the POAG eyes relative to the age-matched healthy eyes, whereas the PLCD was significantly larger. This discrepancy might have been due to the relative irreversibility of peripheral LC displacement, which has been proven by both an experimental animal model and with human glaucomatous eyes. 3,6,7

Among the POAG patients in the current investigation, the males had a significantly larger overall PLCD than did the females. Seo et $a l^{21}$ reported increased anterior LC surface depth in males compared with females in healthy eyes. The PLCD measurement, based on the BMO reference plane, can be influenced by the change of choroidal thickness. Thus, the present finding possibly can be explained by increased choroidal thickness in males than females. ${ }^{22,23}$

Among the other results, the MD of VF showed a significant negative association with overall PLCD in the

Table 3 Comparison of horizontal and vertical peripheral lamina cribrosa depth (PLCD) in primary open-angle glaucoma (POAG) and healthy control eyes

\begin{tabular}{|c|c|c|c|c|c|}
\hline \multirow{2}{*}{ Variable } & \multirow{2}{*}{ Vertical PLCD } & \multirow{2}{*}{ Horizontal PLCD } & \multirow{2}{*}{ P-value } & \multicolumn{2}{|c|}{ Difference (Vertical-Horizontal) } \\
\hline & & & & Mean $\pm S D$ & $95 \% \mathrm{CI}$ \\
\hline POAG ( $n=90$ eyes) & $429.9 \pm 159.0$ & $382.6 \pm 155.3$ & $<0.001^{\mathrm{a}}$ & $47.3 \pm 59.6$ & $34.8-59.8$ \\
\hline Healthy ( $n=90$ eyes) & $357.0 \pm 115.6$ & $339.5 \pm 111.6$ & $0.003^{\mathrm{a}}$ & $17.5 \pm 53.9$ & $6.2-28.8$ \\
\hline
\end{tabular}

Abbreviations: CI, confidence interval; PLCD, peripheral lamina cribrosa depth, defined as vertical distance between most peripheral visible end of anterior lamina cribrosa surface and reference plane of Bruch's membrane opening; POAG, primary open-angle glaucoma.

${ }^{\text {a }}$ Comparison performed using paired $t$-test.

Mean \pm standard deviation, Statistically significant values are shown in bold. 
POAG eyes. Quigley et $a l^{3}$ histologically demonstrated posteriorly displaced laminar insertion in humanautopsied eye with advanced glaucoma. Ren et al ${ }^{24}$ reported that anterior LC surface depth had an agedependent negative association with MD of VF where this effect is weakened as age increases. PLCD might differ from anterior LC surface depth in functional significance, particularly as peripheral LC is where biomechanical stress and vascular supply to the LC meet. Indeed, in consideration of this fact, it could be said that PLCD is a better predictor of glaucoma patients' functional status than anterior LC surface depth, though this remains speculative.

The average RNFL thickness showed significant association with overall PLCD in univariate analysis but not in multivariate analysis. The PLCD measurement was performed at the central three of the five cross-line scans with $0.25-\mathrm{mm}$ spacing at each meridian. This might have excluded the superotemporal and inferotemporal regions from the PLCD measurement, where the RNFL defect most commonly occurs. The nature of the relationship between the RNFL thickness and PLCD should be further evaluated by matching the location of RNFL defect and PLCD measurement via radial scans.

The present study has some limitations. First, one could argue that the healthy eyes have thicker neuroretinal rim width, which hinders the clear visualization of peripheral anterior LC. Thus, the PLCD measurement in healthy eyes might have been biased from this phenomenon. To minimize this, we have matched the disc size between the POAG and healthy eyes. Further, we verified that the distance between the two lines for the PLCD measurement was similar between POAG $(1726.0 \pm 192.3 \mu \mathrm{m})$ and healthy eyes $(1910.5 \pm 873.5 \mu \mathrm{m}$, $P=0.07)$. This might be indirect evidence that the PLCD measurement have been performed within comparable distance from the optic disc border. Second, the majority of the POAG patients had baseline IOP within normal range. And all of the POAG patients were under IOPlowering treatments. The true nature of the relationship between PLCD and IOP can be elucidated from recruiting POAG patients prior to any IOP-lowering treatments, with higher baseline IOP. The present findings should not be generalized to POAG patients with high IOP.

Moreover, they are not reflective of the natural course of POAG.

In conclusion, the present study demonstrated that the PLCD was significantly larger in POAG eyes than in agematched healthy eyes, and that the vertical/horizontal PLCD difference also was larger in POAG eyes. The peripheral LC in the vertical meridian might have increased IOP-related strain (deformation) compared with horizontal meridian in glaucomatous eyes.

\section{Summary}

\section{What was known before}

- Lamina cribrosa (LC) is presumed to be the primary site of axonal injury in glaucoma.

- LC displaces posteriorly in response of intraocular pressure.

- The regional variance in laminar pore and beam size has been suggested to be related with glaucomatous optic nerve damage commonly seen in superior and inferior retinal nerve fibers.

What this study adds

- Peripheral LC depth (PLCD) was significantly larger in primary open-angle glaucoma (POAG) eyes than in agematched healthy eyes.

- The vertical/horizontal PLCD difference also was larger in POAG eyes.

- The peripheral LC in vertical meridian may have increased IOP related strain (deformation) compared with horizontal meridian in glaucomatous eyes.

\section{Conflict of interest}

The authors declare no conflict of interest.

\section{Author contributions}

Design and conduct of the study: YWK, JWJ and KHP, collection of the data: YWK, DWK, JWJ, KHP and DMK, management of the data: YWK, DWK, JWJ and KHP, analysis of the data: YWK, DWK, JWJ and KHP, interpretation of the data: YWK, DWK, JWJ, KHP and DMK, preparation of the manuscript: YWK, DWK, JWJ and KHP, review of the manuscript: YWK, JWJ and KHP, approval of the manuscript: YWK and KHP.

\section{References}

1 Emery JM, Landis D, Paton D, Boniuk M, Craig JM. The lamina cribrosa in normal and glaucomatous human eyes. Trans Am Acad Ophthalmol Otolaryngol 1974; 78(2): OP290-OP297.

2 Quigley HA, Addicks EM. Regional differences in the structure of the lamina cribrosa and their relation to glaucomatous optic nerve damage. Arch Ophthalmol 1981; 99 (1): 137-143.

3 Quigley HA, Addicks EM, Green WR, Maumenee AE. Optic nerve damage in human glaucoma. II. The site of injury and susceptibility to damage. Arch Ophthalmol 1981; 99(4): 635-649.

4 Radius RL. Regional specificity in anatomy at the lamina cribrosa. Arch Ophthalmol 1981; 99(3): 478-480.

5 Radius RL, Gonzales M. Anatomy of the lamina cribrosa in human eyes. Arch Ophthalmol 1981; 99(12): 2159-2162.

6 Quigley HA, Hohman RM, Addicks EM, Massof RW, Green WR. Morphologic changes in the lamina cribrosa 
correlated with neural loss in open-angle glaucoma. Am J Ophthalmol 1983; 95(5): 673-691.

7 Bellezza AJ, Rintalan CJ, Thompson HW, Downs JC, Hart RT, Burgoyne CF. Deformation of the lamina cribrosa and anterior scleral canal wall in early experimental glaucoma. Invest Ophthalmol Vis Sci 2003; 44(2): 623-637.

8 Burgoyne CF, Downs JC, Bellezza AJ, Suh JK, Hart RT. The optic nerve head as a biomechanical structure: a new paradigm for understanding the role of IOP-related stress and strain in the pathophysiology of glaucomatous optic nerve head damage. Prog Retin Eye Res 2005; 24(1): 39-73.

9 Crawford Downs J, Roberts MD, Sigal IA. Glaucomatous cupping of the lamina cribrosa: a review of the evidence for active progressive remodeling as a mechanism. Exp Eye Res 2011; 93(2): 133-140.

10 Sigal IA, Yang H, Roberts MD, Grimm JL, Burgoyne CF, Demirel $\mathrm{S}$ et al. IOP-induced lamina cribrosa deformation and scleral canal expansion: independent or related? Invest Ophthalmol Vis Sci 2011; 52(12): 9023-9032.

11 Yang H, Thompson H, Roberts MD, Sigal IA, Downs JC, Burgoyne CF. Deformation of the early glaucomatous monkey optic nerve head connective tissue after acute IOP elevation in 3-D histomorphometric reconstructions. Invest Ophthalmol Vis Sci 2011; 52(1): 345-363.

12 Yang H, Williams G, Downs JC, Sigal IA, Roberts MD, Thompson $\mathrm{H}$ et al. Posterior (outward) migration of the lamina cribrosa and early cupping in monkey experimental glaucoma. Invest Ophthalmol Vis Sci 2011; 52(10): 7109-7121.

13 Lee KM, Kim TW, Weinreb RN, Lee EJ, Girard MJ, Mari JM Anterior lamina cribrosa insertion in primary open-angle glaucoma patients and healthy subjects. PLoS One 2014; 9 (12): e114935.

14 Dandona L, Quigley HA, Brown AE, Enger C. Quantitative regional structure of the normal human lamina cribrosa. A racial comparison. Arch Ophthalmol 1990; 108(3): 393-398.
15 Jonas JB, Mardin CY, Schlotzer-Schrehardt U, Naumann GO. Morphometry of the human lamina cribrosa surface. Invest Ophthalmol Vis Sci 1991; 32(2): 401-405.

16 Sigal IA, Ethier CR. Biomechanics of the optic nerve head. Exp Eye Res 2009; 88(4): 799-807.

17 Kim TW, Kagemann L, Girard MJ, Strouthidis NG, Sung KR, Leung CK et al. Imaging of the lamina cribrosa in glaucoma: perspectives of pathogenesis and clinical applications. Curr Eye Res 2013; 38(9): 903-909.

18 Park SC. In vivo evaluation of lamina cribrosa deformation in glaucoma. J Glaucoma 2013; 22(Suppl 5): S29-S31.

19 Park SC, Kiumehr S, Teng CC, Tello C, Liebmann JM, Ritch R. Horizontal central ridge of the lamina cribrosa and regional differences in laminar insertion in healthy subjects. Invest Ophthalmol Vis Sci 2012; 53(3): 1610-1616.

20 Furlanetto RL, Park SC, Damle UJ, Sieminski SF, Kung Y, Siegal $\mathrm{N}$ et al. Posterior displacement of the lamina cribrosa in glaucoma: in vivo interindividual and intereye comparisons. Invest Ophthalmol Vis Sci 2013; 54(7): 4836-4842.

21 Seo JH, Kim TW, Weinreb RN. Lamina cribrosa depth in healthy eyes. Invest Ophthalmol Vis Sci 2014; 55(3): 1241-1251.

22 Wei WB, Xu L, Jonas JB, Shao L, Du KF, Wang S et al. Subfoveal choroidal thickness: the Beijing Eye Study. Ophthalmology 2013; 120(1): 175-180.

23 Lee SW, Yu SY, Seo KH, Kim ES, Kwak HW. Diurnal variation in choroidal thickness in relation to sex, axial length, and baseline choroidal thickness in healthy Korean subjects. Retina 2014; 34(2): 385-393.

24 Ren R, Yang H, Gardiner SK, Fortune B, Hardin C, Demirel S et al. Anterior lamina cribrosa surface depth, age, and visual field sensitivity in the Portland Progression Project. Invest Ophthalmol Vis Sci 2014; 55(3): 1531-1539.

Supplementary Information accompanies this paper on Eye website (http://www.nature.com/eye) 